\title{
The simulated in vitro infant gastrointestinal digestion of droplets covered with milk fat globule membrane polar lipids concentrate
}

\author{
Jie Luo, ${ }^{1,2}$ Ziwei Wang, ${ }^{3}$ Yiran Li, ${ }^{3}$ Chong Chen, ${ }^{3}$ Fazheng Ren, ${ }^{1,3}$ and Huiyuan Guo ${ }^{1 *}$ \\ ${ }^{1}$ Beijing Advanced Innovation Center for Food Nutrition and Human Health, College of Food Science and Nutritional Engineering, \\ China Agricultural University, Beijing 100083, China \\ ${ }^{2}$ College of Food Science and Technology, Hunan Agricultural University, Changsha 410004, China \\ ${ }^{3}$ Key Laboratory of Functional Dairy, Co-constructed by Ministry of Education and Beijing Government, China Agricultural University, \\ Beijing 100083, China
}

\section{ABSTRACT}

Milk fat globule membrane polar lipids (MPL) are increasingly used as the surface-active components for emulsions in many infant food products. However, the precise effect of the emulsifier MPL on the digestion of lipids during gastrointestinal digestion has not been elucidated. This study investigated the lipid digestion of droplets covered with MPL with different sizes in a simulated in vitro infant gastrointestinal digestion assay. The well-used surface-active component casein was used as a control. Four types of emulsions were formulated: small and large droplets covered with MPL concentrate (MPL-S and MPL-L, with volumetric means of $0.35 \pm 0.01$ and $4.04 \pm 0.01 \mu \mathrm{m}$, respectively), and small and large droplets covered with casein $(\mathrm{CN}-\mathrm{S}$ and CN-L, with volumetric means of $0.44 \pm 0.01$ and $4.09 \pm$ $0.03 \mu \mathrm{m}$, respectively). The emulsions were subjected to in vitro gastrointestinal digestion using a semidynamic model mimicking infant digestion. Through the determination of particle size evolution, zeta-potential, and microstructure of emulsions, the lipid droplets covered with MPL were found to be more stable than that of the CN-S and CN-L during gastrointestinal digestion. Moreover, although CN-S and CN-L showed a higher initial lipolysis rate at the beginning of gastric digestion, droplets covered by MPL exhibited a significantly higher amount of free fatty acid release during later digestion. The amount of free fatty acid release of the emulsions in both gastric and intestinal digestion could be generally classified as MPL-S $\geq$ MPL-L $>$ CN-S > CN-L. Our study highlights the crucial role of MPL in the efficient digestion of emulsions and brings new insight for the design of infant food products.

Key words: in vitro gastrointestinal digestion, infant, milk polar lipids, fat globules size

Received May 11, 2018.

Accepted December 7, 2018.

*Corresponding author: guohuiyuan@cau.edu.cn

\section{INTRODUCTION}

Proteins and low-molecular-weight surfactants are widely used as the surface-active components for emulsions in many food products (Cornec et al., 2010). The formation and stabilization of the emulsions strongly depend on the interfacial properties of these surfaceactive components. Recently, low-molecular-weight surfactant milk fat globule membrane polar lipids (MPL) have been a focus of interest.

Membrane polar lipids are mainly located in the highly structured 3-layered milk fat globule membrane (MFGM) surrounding the milk fat globules (Lopez, 2011); MPL can prevent the coalescence of fat globules by exerting a repulsive force with the ionogenic groups (Shimizu et al., 1980). Moreover, specific MPL, such as sphingomyelin (SM), have been shown to have important biological functions, such as in gut maturation and myelination of the developing central nervous system of newborns (Spitsberg, 2005). Therefore, the application of MPL as emulsifiers in infant formula foods to improve several growth and developmental outcomes has generated a great deal of interest (Oosting et al., 2012; Gallier et al., 2015; Schipper et al., 2016).

The specific types of emulsifiers greatly affect the interfacial characteristics of emulsions and thereby affect their susceptibility to digestive hydrolysis during digestion (Michalski, 2009; Golding and Wooster, 2010; Golding et al., 2011). Recent studies have compared the in vitro gastrointestinal digestion of native milk fat globules that are mainly covered by MPL and membrane-specific proteins with processed fat globules (by homogenization and heat treatment) that are mainly covered by casein and whey protein. The results showed that native milk fat droplets show faster lipolysis and gastric emptying than the processed fat droplets (Bourlieu et al., 2015; de Oliveira et al., 2017); however, the native MFGM was composed of nonsingular interfacial components. In addition to the MPL, MFGM proteins can also be used as emulsifying agents due to their 
amphiphilic nature (Vanderghem et al., 2010) and are involved in the lipid digestion process (Vanderghem et al., 2011; Ye et al., 2011). Therefore, the precise effect of emulsifier MPL on the digestion of lipids remains unknown.

In addition to the interfacial composition, the size of emulsion droplets also exerts a great influence on the digestion of lipids. Compared with large fat globules, smaller fat globules have increased surface area for lipase anchoring, which contributes to their faster digestion (Singh and Gallier, 2017). A comparison of the digestion rate between homogenized milk and human milk showed that, despite the 25 -fold larger available surface area of fat globules in homogenized milk, ability for the hydrolysis of milk triacylglycerols increased only 2-fold from homogenized milk than in human milk (Berton et al., 2012). This indicated that the interfacial composition and size of emulsion droplets synthetically affect the lipolysis of lipids. However, no research has systematically compared the lipolysis process of lipid droplets covered with MPL with different sizes during gastrointestinal digestion.

As MPL are increasingly used in infant formula, and because the digestion process in infants greatly differs from that in adults (Bourlieu et al., 2014), we investigated the lipid digestion of droplets covered with MPL of different sizes in gastrointestinal conditions that mimicked those reported in newborns. The findings of the study could contribute to a better understanding of the effect of interfacial component MPL as well as their droplet sizes on the neonatal gastrointestinal digestion of lipids and be useful to the infant food industry.

\section{MATERIALS AND METHODS}

\section{Materials}

Infant vegetable oil (Infat) was purchased from Advanced Lipids (Karlshamn, Sweden) and butter milk powder was obtained from Fonterra Co-operative Group (Auckland, New Zealand). Pepsin powder from porcine gastric mucosa (catalog no. P7000; $\geq 250$ units/ $\mathrm{mg}$ of solid) and fungal lipase from Rhizopus oryzae (catalog no. 62305, Sigma-Aldrich, St. Louis, MO; 50 units $/ \mathrm{mg}$ of solid, stable in the range $\mathrm{pH} 4.5-7.5$, sn-1(3) stereospecific enzyme) were used to simulate the stomach environment. Pancreatin from porcine pancreas (catalog no. P7545; 8× USP specification), lipase from porcine pancreas (catalog no. L3126; Type II, 100-500 units/mg of protein using olive oil), and bile salt extract (catalog no. 48305) were obtained from Sigma-Aldrich (St. Louis, MO) and used to simulate the intestinal digestion environment. Unless otherwise stated, chemicals were from Sigma-Aldrich.

\section{Preparation of 4 Kinds of Emulsions}

Total lipids were extracted from the butter milk powder as the MPL concentrate according to a previously published method (Phan et al., 2016), except the extracting agent was dichloromethane/methanol. The content of polar lipids in the concentrate was determined using HPLC with an evaporative light scattering detector, as previously described (Luo et al., 2018). The MPL concentrate comprised about $16 \%$ (wt/wt) of the total lipids, with $3.68 \pm 0.02 \%$ phosphatidylethanolamine (PE), $6.30 \pm 0.10 \%$ phosphatidylcholine (PC), and $5.29 \pm 0.06 \%$ SM. The rest of total lipids was mainly triglycerides and included trace amounts of other minor neutral lipids, such as cholesterol and its esters. As a large quantity of MPL were needed to prepare sufficient emulsions for digestion tests and extracting MPL from the butter milk powder required much work, we did not further distinguish the MPL fraction from the neutral lipids.

The well-used surface-active component casein was used as a control. Four kinds of oil-in-water emulsions were prepared by mixing infant oil $(2.5 \%$, wt/wt) as core material with MPL concentrate $(4 \%$, wt/wt) or sodium caseinate solution ( $1 \%$, wt/wt) as surface material. The concentrations were chosen based on analysis of the optimum stability of the emulsions determined by Turbiscan (Formulaction, Toulouse, France; data not shown). A 2-stage high-pressure homogenizer at 50/5 MPa (NS1001L2K, GEA Niro Soavi, Parma, Italy) was used to prepare emulsions with large droplets and at $50 / 5 \mathrm{MPa}$ to prepare emulsions with small droplets. The small and large droplets covered with MPL concentrate were named MPL-S and MPL-L, respectively, and the small and large globules covered with casein were named $\mathbf{C N}-\mathbf{S}$ and $\mathbf{C N}-\mathbf{L}$, respectively. If the reagents were not used within the same day, sodium azide $(0.02 \%, \mathrm{wt} / \mathrm{wt})$ was added and the emulsions were kept at room temperature for a maximum of $2 \mathrm{~d}$.

\section{Characterization of Emulsions}

Particle Size Measurements. The size distribution of emulsions was measured by laser light scattering using a Mastersizer 3000 laser diffraction particle size analyzer (Malvern Instruments Ltd., Malvern, United Kingdom) with 2 laser sources, allowing the characterization of micronic as well as submicronic populations. The refractive indices used were 1.333 and 1.460 at 466 $\mathrm{nm}$ for water and fat droplets, respectively. A pinch of samples was added to $500 \mathrm{~mL}$ of distilled water in the measurement cell of the apparatus to reach 10 to $20 \%$ obscuration. The average volume-weighted diameter $\left(\mathbf{D}_{4,3}\right)$ was determined as 


$$
\mathrm{D}_{4,3}=\Sigma n i d i^{4} / \Sigma n i d i^{3},
$$

where $n i$ is the number of globules in a size class of diameter $d i$.

Observation of Emulsions by 3-Dimensional Structured Illumination Fluorescence Light Microscopy. Emulsions were centrifuged at $4,000 \times g$ for $5 \mathrm{~min}$ at $4^{\circ} \mathrm{C}$ and the top cream layer was retained; $0.35 \mathrm{~g}$ of cream was then gently mixed with $5 \mathrm{~mL}$ of Milli-Q water from a Milli-Q water purification system (Millipore, Bedford, MA). The fluorescent probe $\mathrm{N}$ (lissamine rhodamine B sulfonyl) dioleoylphosphatidylethanolamine (Rd-DOPE, excitation laser $=543 \mathrm{~nm}$; Avanti Polar Lipids Inc., Birmingham, UK) was used at a concentration of $1 \mathrm{mg} / \mathrm{mL}$ in chloroform to label the phospholipids in the interface of droplets covered by MPL. Fast Green (Sigma-Aldrich) was used at a concentration of $1 \mathrm{mg} / \mathrm{mL}$ in Milli-Q to label the protein in the interface of droplets covered by casein. Twenty-five microliters of Rd-DOPE and $100 \mu \mathrm{L}$ of Fast Green were mixed with $200 \mu \mathrm{L}$ of emulsions and the samples were kept in the dark at room temperature for $1 \mathrm{~h}$. Lowmelting-point agarose was prepared at $1 \%$ (wt/wt) and slowly mixed with the milk at a ratio of $2: 1$, and the samples were observed using 3-dimensional structured illumination fluorescence light microscopy (3D-SIM). The 3D-SIM images of emulsions were acquired at room temperature $\left(25^{\circ} \mathrm{C}\right)$ on the Delta Vision OMX V3 imaging system (Applied Precision, GE Healthcare, Chicago, IL) with a $100 \times 1.4$ oil objective (UPLSAPO, Olympus Corp., Tokyo, Japan), solid-state multimode lasers (488 and $561 \mathrm{~nm}$ ), and electron-multiplying charge-coupled device cameras (Evolve $512 \times 512$, Photometrics, Tuscon, AZ). Serial Z-stack sectioning was performed at 250-nm intervals for conventional mode.

Lipids Composition Determination. Fatty acid compositions of droplets from different emulsions were analyzed by GC, as described in Chinese Standard (2016). Polar lipids content of droplets was determined as previously described.

Simulated In Vitro Infant Gastric Digestion. To mimic the physiological processes of infant gastric digestion, $\mathrm{pH}$, simulated gastric fluid composition, and the concentration of digestive enzymes were adjusted according to the in vivo data in previous reports (Gallier et al., 2012; Bourlieu et al., 2015). The pH-stat device was programmed to establish 4 subsequent stages at pH 6.5 (0-30 min), 6.0 (30-60 min), 5.5 (60-90 min), and 5.0 (90-120 min; Roman et al., 2007; Bourlieu et al., 2014). At the beginning of each stage, the emulsion $\mathrm{pH}$ was adjusted to the setting value by adding the simulated gastric fluid with $0.4 \mathrm{mg} / \mathrm{mL}$ of pepsin and $0.8667 \mathrm{mg} / \mathrm{mL}$ of lipase. The temperature and the stirring speed were maintained at $37^{\circ} \mathrm{C}$ and $370 \mathrm{rpm}$, respectively, during the entire digestion using the stirring apparatus (HJ-6A, Ronghua, Jiangsu, China).

Intestinal Digestion. After $120 \mathrm{~min}$ of gastric digestion, the in vitro infant intestinal digestion was carried out by mixing the digested emulsions with the simulated intestine solution (1:1), adjusting the $\mathrm{pH}$ to 7.0 , and adding bile extract $(5 \mathrm{mg} / \mathrm{mL})$. The simulated intestine solution was prepared to closely mimic the physiological processes of infant intestinal digestion (Bourlieu et al., 2015). The mixture was incubated at $37^{\circ} \mathrm{C}$ in a shaking water bath (370 rpm). Once the digestion started, the mixed enzyme solution (pancreatin and pancreatic lipase, $1.6 \mathrm{mg} / \mathrm{mL}$ each) was mixed with the diluted emulsions.

Release of Free Fatty Acids. The amount of $\mathrm{NaOH}$ required to maintain equilibrium was used to calculate the concentration of titratable free fatty acids (FFA) released during the simulated in vitro infant gastrointestinal digestion. Because the MPL concentrate extracted from buttermilk contained about $16 \%$ MPL and $84 \%$ neutral lipids (mainly triglyceride), we found differences in the original triglyceride concentration between the MPL and CN groups (i.e., 5.86 vs. $2.5 \%$, respectively). Therefore, the normalized FFA of different emulsions as a function of digestion time is presented. The normalized FFA was determined as

$$
\text { Normalized FFA }=O_{F F A} / T_{c},
$$

where $O_{F F A}$ is the measured values of FFA amount and $T_{c}$ is the triglyceride concentration.

\section{Characterization of Emulsions During Digestion}

At various times during both gastric and intestine digestion $(0,15,30,45,60,75,90,105$, and $120 \mathrm{~min})$, aliquots $(1.5 \mathrm{~mL})$ were collected and the reactions were terminated by heating at $80^{\circ} \mathrm{C}$ for $30 \mathrm{~min}$. The samples were then subjected to various analyses.

Particle Size and Zeta-Potential Measurements. Particle size measurements were determined as described earlier in the text.

The apparent zeta-potential of emulsions was measured at $25^{\circ} \mathrm{C}$ using a Malvern Zetasizer Nano ZS instrument (Malvern Instruments Ltd.) equipped with palladium electrodes and an avalanche photodiode detector (enhancing sensitivity). Typical dilutions were from 4 to $8 \times 10^{-3}$ and measurements were performed rapidly after diluting the sample 1,000:1 with Milli-Q water.

Morphology Observation. The morphologies of the emulsions during digestion were observed with optical microscopy (Axiovert A1, Zeiss Axioscope, Zeiss, Oberkochen, Germany). Ten microliters of emulsion 


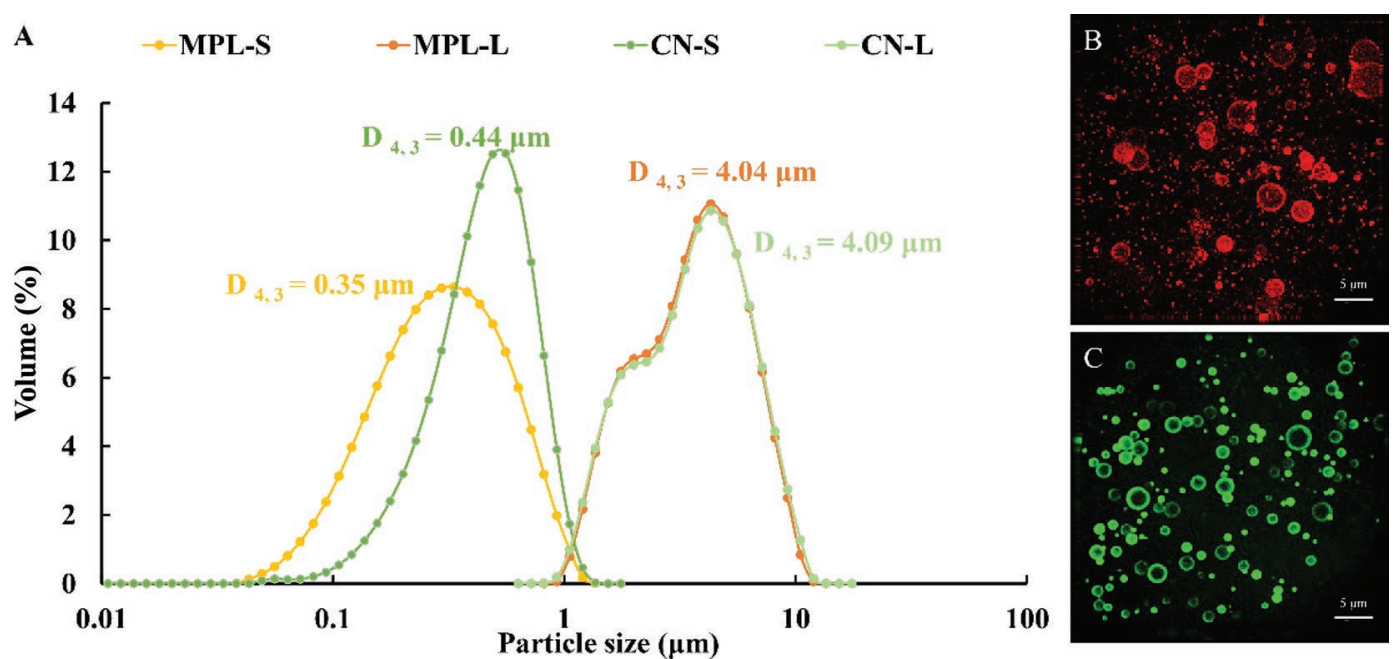

Figure 1. Particle size distributions (A) and 3-dimensional structured illumination fluorescence light microscopy images (B and C) of emulsions covered by milk polar lipids and casein. Emulsions were stained (B) with $N$-(lissamine rhodamine B sulfonyl) dioleoylphosphatidylethanolamine (Rd-DOPE, Avanti Polar Lipids Inc., Birmingham, UK; red) and (C) with Fast Green (Sigma-Aldrich, St. Louis, MO; green). Scale bars $=5 \mu \mathrm{m}$. MPL-S and MPL-L indicate small and large globules covered with milk fat globule membrane polar lipids; CN-S and CN-L indicate small and large globules covered with casein, respectively. $\mathrm{D}_{4,3}=$ volume-weighted diameter.

was placed on a microscope glass slide and covered with a glass coverslip; all samples were examined with a $20 \times$ magnification lens.

\section{Statistical Analyses}

All measurements were performed in triplicate and expressed as mean and standard deviation. The data for digestion were analyzed by 2-way ANOVA with emulsion group, digestion time, and the interaction between group and time as the model variables (with Tukey test) using SPSS software (version 22.0, IBM, Armonk, NY). Other data were analyzed by one-way ANOVA (with Duncan's multiple range method). The level for statistical significance was set at $P<0.05$.

\section{RESULTS}

\section{Characteristics of the Emulsions Prepared}

Four types of emulsions were formulated: small and large droplets covered with MPL (MPL-S and MPL-L, respectively) and small and large droplets covered with casein (CN-S and CN-L, respectively). The particle size distributions of emulsions prepared with different emulsifiers of different sizes are shown in Figure 1A. All 4 emulsions showed a unimodal and concentrated particle size distribution, indicating that emulsions had been effectively prepared. The particle size distribution curves of MPL-L and CN-L were almost overlapping, with the $\mathrm{D}_{4,3}$ of MPL-L $(4.04 \pm 0.01 \mu \mathrm{m})$ and CN-L $(4.09 \pm 0.03$ $\mu \mathrm{m})$ consistent with that of the fat globules in mature human milk ( $\sim 4 \mu \mathrm{m}$; Li et al., 2010). Moreover, the $\mathrm{D}_{4,3}$ of MPL-S $(0.35 \pm 0.01 \mu \mathrm{m})$ and CN-S $(0.44 \pm 0.01 \mu \mathrm{m})$ were similar to the size of fat globules in the current infant milk formula $(\sim 0.4 \mu \mathrm{m}$; Gallier et al., 2015$)$. We also performed 3D-SIM in combination with fluorescent probes to confirm the interfacial composition of the emulsions. The MPL-L droplets showed polar lipids at their surface as a thin layer or occasionally as thick patches (Figure 1B), whereas CN-L droplets showed protein at their surface as a thin layer (Figure 1C).

Moreover, the fatty acid and the polar lipid composition of droplets prepared were determined and shown in Figure 2 and Table 1, respectively. The results indicated that the fatty acid composition of droplets did not differ within the MPL and CN groups (Figure 2). In addition, the polar lipid composition was not significantly different between MPL-S and MPL-L (Table 1, $P>0.05)$.

\section{Characteristics of the Emulsions During Digestion}

Evolution of Particle Size Distribution. We first monitored the evolution of particle size distribution and the $\mathrm{D}_{4,3}$ of fat globules during the simulated in vitro infant gastrointestinal digestion (Figure 3, Table 2). At the beginning of the in vitro digestion, all groups showed a unimodal distribution, but the distribution became bimodal and multimodal as the digestion progressed. During the in vitro gastric digestion, the droplet size in both the MPL and CN groups significantly increased $(P<0.05)$, indicating that the structure of the lipid droplet was destabilized and the 
Table 1. Concentration of polar lipids ${ }^{1}$ in fat droplets covered by milk fat globule membrane polar lipids (MPL) concentrate ${ }^{2}$

\begin{tabular}{lccc}
\hline Sample $^{3}$ & PE (\%) & PC (\%) & SM (\%) \\
\hline MPL-S & $1.98 \pm 0.06^{\mathrm{a}}$ & $3.51 \pm 0.11^{\mathrm{a}}$ & $2.82 \pm 0.08^{\mathrm{a}}$ \\
MPL-L & $2.06 \pm 0.04^{\mathrm{a}}$ & $3.47 \pm 0.04^{\mathrm{a}}$ & $2.91 \pm 0.07^{\mathrm{a}}$ \\
\hline
\end{tabular}

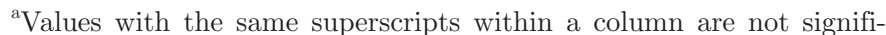
cantly different $(P>0.05)$.

${ }^{1} \mathrm{PE}=$ phosphatidylethanolamine; $\mathrm{PC}=$ phosphatidylcholine; $\mathrm{SM}=$ sphingomyelin.

${ }^{2}$ Results are mean $\pm \mathrm{SD}(\mathrm{n}=3)$.

${ }^{3} \mathrm{MPL}-\mathrm{S}$ and MPL-L $=$ small and large globules covered with milk fat globule MPL, respectively;

droplets gradually aggregated. As the gastric digestion proceed (60-120 $\mathrm{min})$, the particles covered by casein showed a much more dramatic aggregation than that of MPL (tens to hundreds fold vs. several fold larger than the original size). In addition, the MPL-S group remained smaller than the MPL-L group throughout the gastric digestion; however, the $\mathrm{CN}-\mathrm{S}$ showed a significantly larger size than that of CN-L after $120 \mathrm{~min}$ of gastric digestion $(15.78 \pm 0.10$ vs. $9.21 \pm 0.10 \mu \mathrm{m}$, Table 2; $P<0.05)$.

During the intestinal digestion period, the aggregated particles were mixed with the simulated intestinal fluid and the $\mathrm{pH}$ was back up to 7.0 with the bile salt. Upon mixing with the simulated intestinal fluid, the size of droplets covered by casein immediately decreased, mainly due to the dissociation and redispersal of casein micelles bonding under a higher $\mathrm{pH}$ and the interface displacement of bile salts (Gallier et al., 2012). In contrast, the size of droplets covered by MPL first significantly increased, with the reducing stability caused by the bile salt as well as the lipolysis products. As the digestion proceed, the size of droplets covered by MPL gradually decreased as the pancreatic lipase worked. Aggregates formed during intestinal digestion were larger in the large droplets than the small ones.

Zeta-Potential. We next evaluated the zetapotential of droplets in different emulsions during the simulated in vitro infant gastrointestinal digestion (Figure 4). Zeta-potential is an important index that indicates the stability of emulsions (Michalski et al., 2002) and indirectly reflects changes in the interfacial composition of droplets (Gallier et al., 2012). After 30 min of simulated infant digestion, droplets of MPL carried a greater negative charge compared with $\mathrm{CN}$. This may be because the $\mathrm{pH}$ decreased as the emulsions were exposed to stomach acid and getting close to the isoelectric point of casein. Therefore, the absolute zeta-potentials of the $\mathrm{CN}$ groups were significantly reduced compared with the MPL groups $(P<0.05)$. In addition, except for that of $\mathrm{CN}$ groups determined after 120 min gastric digestion, the absolute values of zeta-potentials of small droplets were larger than those of the large ones within each group.

In the simulated intestinal digestion, the pregastric digested emulsions were mixed with simulated intestinal fluid and bile extracts at $\mathrm{pH}$ 7.0. The charge of the droplet surface turned negative as the $\mathrm{pH}$ shifted above the isoelectric point of the proteins and peptides and the bile salts displaced the MPL, casein, and peptides from the interface of the globules, as bile salts are highly negatively charged at pH 7.0 (Aken et al., 2010). Therefore, the interface of the droplets was altered and the zeta-potential of all groups determined at the beginning of intestinal digestion were significantly lower than those determined at the end of gastric digestion, as seen in Figure 4B. During the later intestinal digestion stage, the zeta-potential of all emulsion droplets

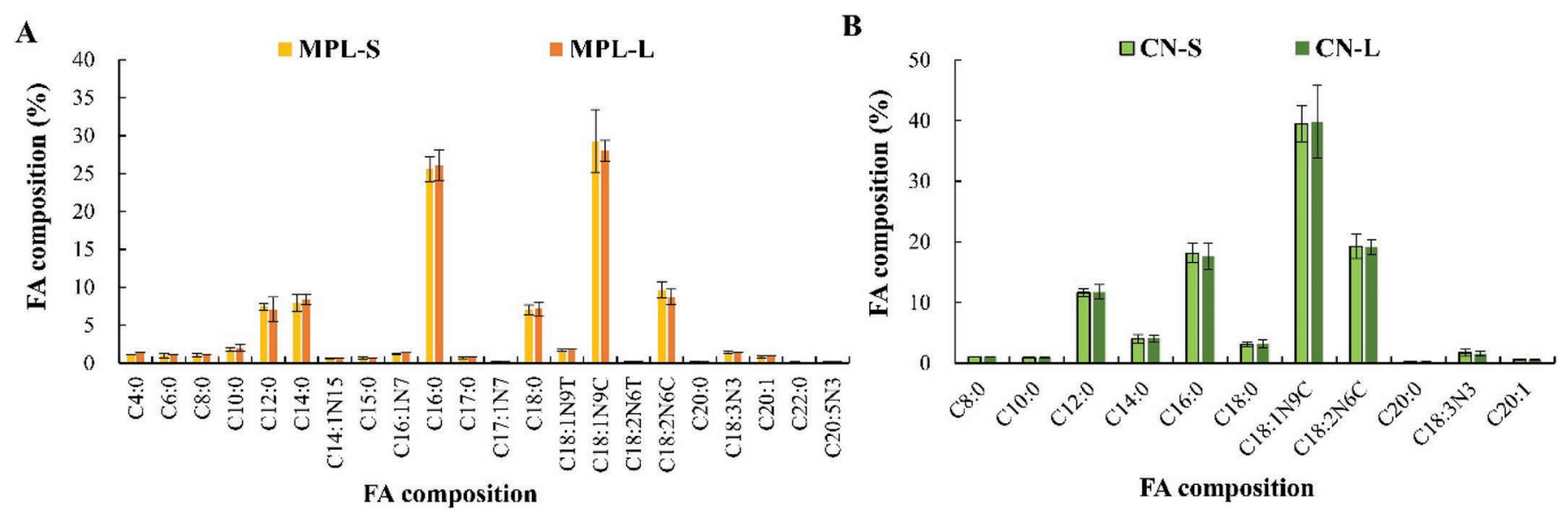

Figure 2. Fatty acid (FA) composition of droplets covered by milk polar lipids and casein. MPL-S and MPL-L indicate small and large globules covered with milk fat globule membrane polar lipids and CN-S and CN-L indicate small and large globules covered with casein, respectively. $\mathrm{T}=$ trans $; \mathrm{C}=$ cis. Error bars represent $\mathrm{SD}$ of triplicate experiments. 

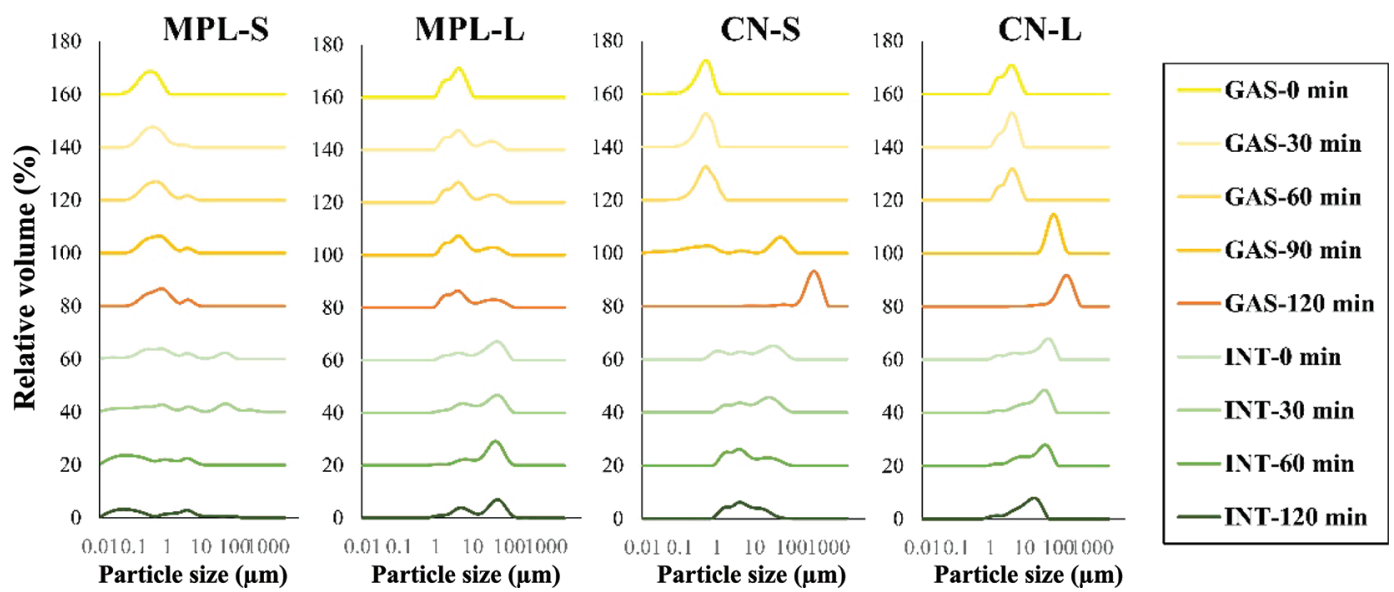

Figure 3. Evolution of particle size distribution during in vitro infant gastric (GAS) digestion and intestinal (INT) digestion. MPL-S and MPL-L indicate small and large globules covered with milk fat globule membrane polar lipids, and CN-S and CN-L indicate small and large globules covered with casein, respectively.

slightly changed. After 30 min of intestinal digestion, the MPL-S carried significantly more negative charge compared with other groups; no significant difference was found among other globules (Figure 4B).

Microstructural Changes. The microstructural changes of emulsions during gastrointestinal digestion were followed by optical microscopy (Figure 5). An obvious aggregation of casein was observed in the CN groups after 120 min of gastric digestion, whereas the fat globules of the MPL groups remained dispersed in the emulsions up to 120 min into gastric digestion, indicating more stabilized globules throughout gastric digestion. During the intestinal digestion, the large aggregates formed in CN-S and CN-L groups at the gastric phase were dissociated and redispersed, as shown by the reformed droplets of smaller sizes in Figures $5 \mathrm{~K}$ and 5O. Indeed, visual observation of the $\mathrm{CN}$ samples after the intestine phase indicated that the samples did not contain any visibly large aggregates or sediments. In contrast, some aggregated droplets were found in the MPL-L sample after $60 \mathrm{~min}$ of intestinal digestion. The aggregates observed in this sample may have been aggregated lipid droplets or micelles and vesicles assembled from bile salts, phospholipids, FFA, and monoacylglycerols (Maldonado-Valderrama et al., 2011).

FFA Release. Development of the normalized FFA from different emulsions as a function of digestion time is presented in Figure 6. The results indicated a significant difference existed in the amount of FFA release as well as the lipid hydrolysis rates between the MPL and CN groups during gastric digestion $(P<0.05)$. During the first $15 \mathrm{~min}$ of gastric digestion, the amount of

Table 2. Evolution of volume-weighted diameter $\left(\mathrm{D}_{4,3}, \mu \mathrm{m}\right)$ of droplets ${ }^{1}$ from 4 emulsions during simulated in vitro infant gastric and intestinal digestion ${ }^{2}$

\begin{tabular}{|c|c|c|c|c|c|}
\hline $\mathrm{D}_{4,3}(\mu \mathrm{m})$ & $\begin{array}{l}\text { Time } \\
(\min )\end{array}$ & MPL-S & MPL-L & $\mathrm{CN}-\mathrm{S}$ & CN-L \\
\hline \multirow[t]{5}{*}{ Gastric } & 0 & $0.35 \pm 0.01^{\mathrm{a}, \mathrm{A}}$ & $4.04 \pm 0.01^{\mathrm{a}, \mathrm{B}}$ & $0.44 \pm 0.01^{\mathrm{a}, \mathrm{A}}$ & $4.09 \pm 0.03^{\mathrm{a}, \mathrm{B}}$ \\
\hline & 30 & $0.59 \pm 0.02^{\mathrm{b}, \mathrm{A}}$ & $12.80 \pm 0.33^{\mathrm{b}, \mathrm{C}}$ & $0.49 \pm 0.01^{\mathrm{a}, \mathrm{A}}$ & $4.29 \pm 0.00^{\mathrm{a}, \mathrm{B}}$ \\
\hline & 60 & $0.88 \pm 0.00^{\mathrm{c}, \mathrm{B}}$ & $13.53 \pm 0.37^{\mathrm{bc}, \mathrm{D}}$ & $0.48 \pm 0.01^{\mathrm{a}, \mathrm{A}}$ & $4.43 \pm 0.03^{\mathrm{a}, \mathrm{C}}$ \\
\hline & 90 & $0.98 \pm 0.00^{\mathrm{d}, \mathrm{A}}$ & $14.03 \pm 0.66^{\mathrm{c}, \mathrm{B}}$ & $27.83 \pm 0.45^{\mathrm{b}, \mathrm{C}}$ & $82.60 \pm 1.59^{\mathrm{b}, \mathrm{D}}$ \\
\hline & 120 & $1.17 \pm 0.06^{\mathrm{e}, \mathrm{A}}$ & $17.33 \pm 0.50^{\mathrm{d}, \mathrm{B}}$ & $417.25 \pm 3.77^{\mathrm{c}, \mathrm{D}}$ & $179.00 \pm 4.90^{\mathrm{c}, \mathrm{C}}$ \\
\hline \multirow{4}{*}{ Intestinal } & 0 & $10.09 \pm 0.54^{\mathrm{a}, \mathrm{A}}$ & $32.70 \pm 0.83^{\mathrm{b}, \mathrm{C}}$ & $20.78 \pm 0.17^{\mathrm{d}, \mathrm{B}}$ & $34.38 \pm 0.53^{\mathrm{c}, \mathrm{D}}$ \\
\hline & 30 & $37.60 \pm 8.66^{\mathrm{b}, \mathrm{B}}$ & $31.65 \pm 0.25^{\mathrm{b}, \mathrm{B}}$ & $19.35 \pm 0.29^{\mathrm{c}, \mathrm{A}}$ & $29.40 \pm 0.24^{\mathrm{b}, \mathrm{B}}$ \\
\hline & 60 & $1.55 \pm 0.07^{\mathrm{a}, \mathrm{A}}$ & $36.65 \pm 0.86^{\mathrm{c}, \mathrm{D}}$ & $12.00 \pm 0.29^{\mathrm{b}, \mathrm{B}}$ & $29.35 \pm 0.31^{\mathrm{b}, \mathrm{C}}$ \\
\hline & 120 & $2.94 \pm 0.76^{\mathrm{a}, \mathrm{A}}$ & $25.88 \pm 0.97^{\mathrm{a}, \mathrm{D}}$ & $9.21 \pm 0.10^{\mathrm{a}, \mathrm{B}}$ & $15.78 \pm 0.10^{\mathrm{a}, \mathrm{C}}$ \\
\hline
\end{tabular}

${ }^{\mathrm{a} e}$ Means with different lowercase letters are significantly different within the same column for each digestion period $(P<0.05)$.

${ }^{\mathrm{A}-\mathrm{D}}$ Means with different uppercase letters are significantly different within the same line $(P<0.05)$.

${ }^{1}$ MPL-S and MPL-L = small and large droplets covered with milk fat globule membrane phospholipid, respectively; CN-S and CN-L = small and large droplets covered with casein, respectively.

${ }^{2}$ Data are the means and SD of 3 different experiments. 
A

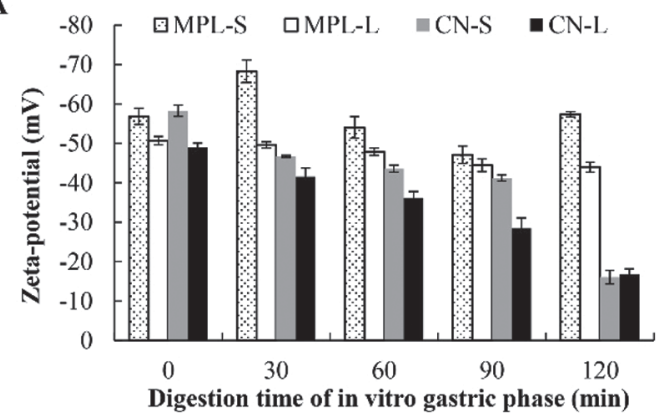

B

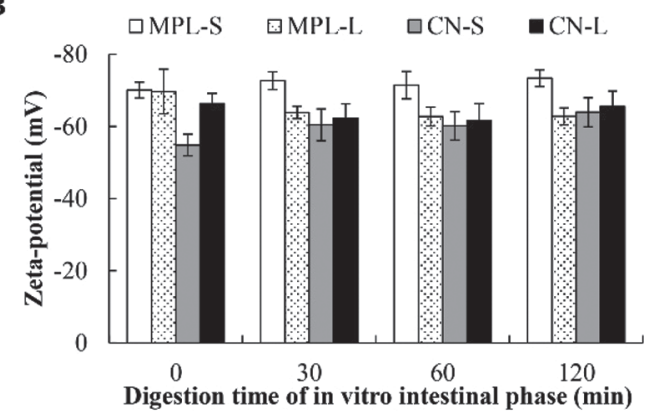

Figure 4. Zeta-potential of droplets from different emulsions during in vitro gastric (A) and intestinal digestion (B). MPL-S and MPL-L indicate small and large globules covered with milk fat globule membrane polar lipids, and CN-S and CN-L indicate small and large globules covered with casein, respectively. Error bars represent the SD of triplicate experiments.

FFA released and the lipolysis rates of the CN groups were significantly higher than those of the MPL groups, and the trend could be classified as $\mathrm{CN}-\mathrm{S}>\mathrm{CN}-\mathrm{L}>$ MPL-S $\geq$ MPL-L. However, the lag phase of hydrolysis in the CN samples occurred after 30 min gastric digestion, whereas the amount of FFA release continually increased during the entire simulated gastric stage. The FFA release amount at the end of gastric digestion could be classified as MPL-S $\geq$ MPL-L $>$ CN-S $\gg$ CN-L.

During the simulated intestinal digestion phase, the amount of FFA release of all emulsions increased steadily during the first $90 \mathrm{~min}$ and then leveled off to a plateau toward the end of digestion (Figure $6 \mathrm{~B}$ ). The initial lipolysis rate and the FFA release amount of emulsions was established as MPL-S > MPL-L > CN-S > CN-L, but the differences between different sizes narrowed with the digestion time.

\section{DISCUSSION}

The digestion process in infants differs from that in adults because of the immaturity of the digestive tract, which shows lower enzyme activity, gastric and pancreatic juice secretion, bile salt concentration, and gastric motility at birth (Bourlieu et al., 2014). To investigate the lipid digestion of droplets covered with MPL of different sizes in a simulated in vitro infant gastrointestinal digestion, 4 emulsions were prepared for the digestion test. Using particle size determination and a fluorescent probe, the 4 emulsions were assigned as either large droplets covered by MPL or casein as control (MPL-L and CN-L), mimicking the size of globules in the human milk, or small droplets covered by MPL or casein (MPL-S and CN-S), mimicking the size of globules in the infant milk formula. Moreover, by analyzing the fatty acid and polar lipid composition of droplets prepared, we confirmed the lipid compositions were not significantly different between small and large droplets within the MPL and CN groups.

\section{Effect of Interfacial Components on the In Vitro Infant Gastrointestinal Digestion}

Gastric Digestion. Triglyceride digestion is initiated by gastric lipase and the adsorption of gastric lipase onto the emulsion interface is $\mathrm{pH}$-dependent and driven by hydrophobic and electrostatic interactions (Chahinian et al., 2006; Bénarouche et al., 2013). Infants have significant higher intragastric $\mathrm{pH}$ values when compared with that of the adults. The pH decrease in the infant's stomach is much slower and the $\mathrm{pH}$ value stays above 5.0 after gastric digestion for $120 \mathrm{~min}$, whereas that in an adult's stomach would be below 3.0 (Bourlieu et al., 2015). As the optimal $\mathrm{pH}$ of human gastric lipase is around 5.6, gastric digestion plays a central role in lipid digestion in infants (Sams et al., 2016).

At the beginning of gastric digestion, due to the existence of both hydrophobic interaction and electronegativity in droplets covered with casein, the gastric lipase would be adsorbed faster onto the emulsion interface covered by caseins than the MPL. This may explain why the initial lipolysis rates of the $\mathrm{CN}$ groups were significantly higher than those of the MPL groups. As the gastric digestion proceeded, the electronegativity of $\mathrm{CN}$ groups was greatly reduced due to the decreasing $\mathrm{pH}$, as could be seen from their zeta-potential values. In contrast, the electrostatic interactions between MPL and gastric lipases were favored at a low $\mathrm{pH}$ (Bourlieu et al., 2016). Therefore, the FFA release of the MPL groups continually increased and finally showed a higher amount than those of the CN groups.

Droplets covered by MPL were significantly more stable than that of the $\mathrm{CN}$ groups during gastric digestion. The presence of enzymes, the decrease of $\mathrm{pH}$, and the hydrolysis of interfacial proteins could cause 


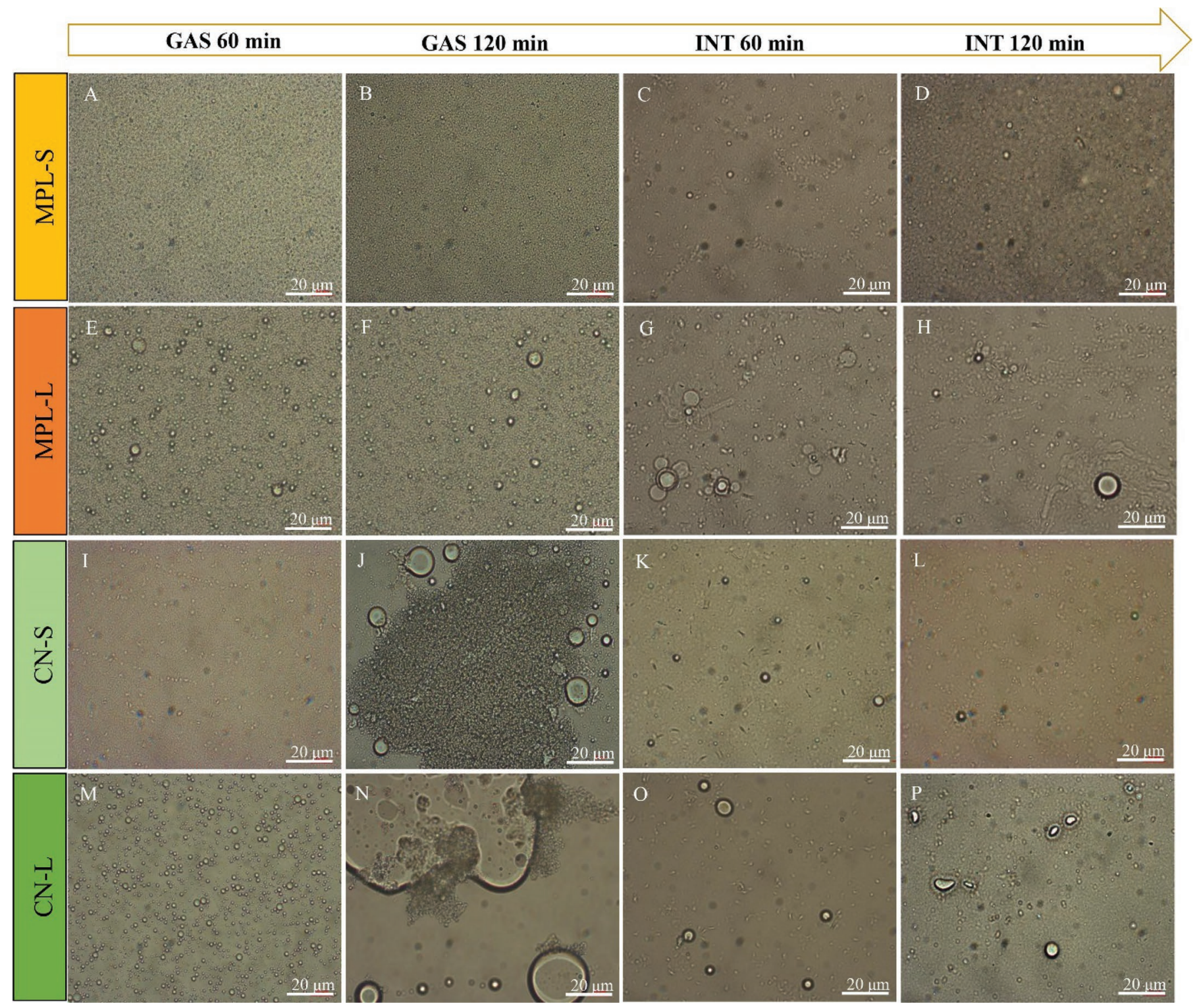

Figure 5. Morphologies of the emulsions during in vitro infant gastric (GAS) and intestinal (INT) digestion. MPL-S and MPL-L indicate small and large globules covered with milk fat globule membrane polar lipids, and CN-S and CN-L indicate small and large globules covered with casein, respectively. Scale bars $=20 \mu \mathrm{m}$.

the aggregation of the globules (Gallier et al., 2013a,b). As the gastric digestion proceeded, the absolute zetapotential values of droplets covered by casein were significantly reduced, indicating greatly reduced stability of these droplets. As can be seen from their particle size and microstructure, a large number of aggregated fat globules occurred in the CN groups at the end of gastric digestion. The difference in destabilization rate and aggregation level of droplets might also affect gastric lipid bioaccessibility and contribute to the difference in the lipolysis rate and gastric emptying (Gallier et al., 2017).
Bourlieu et al. (2015) also reported that most of the lipid hydrolysis occurred during the first $30 \mathrm{~min}$ of gastric digestion and the rate of lipolysis was significantly higher for the processed milk fat globules covered with milk protein than that of the native milk fat globules covered by MPL and membrane-specific protein. However, those authors considered that the initial lipolysis rate difference was mainly governed by the size of the droplets rather than the interfacial components; once the specific surface was normalized, then the native and processed milk fat globules showed no difference in lipolysis rate (Bourlieu et al., 2015). The contradictory 
conclusions may be attributed to the different droplet sizes of emulsions used in their study. As the sizes of droplets were quite different, the researchers could not directly compare the effect of the interfacial composition on the lipolysis of emulsions. Consequently, they compared droplets by normalizing the specific surface area of droplets of different sizes. However, as discussed earlier, the lipolysis ability dose not correspond linearly to the available surface area of fat droplets (Berton et al., 2012). The interfacial composition and size of emulsion droplets synthetically affect the lipolysis of lipids. Moreover, whey proteins, such as $\beta-\mathrm{LG}$ and membrane-specific protein, have been shown to be involved during the lipid digestion process (Mandalari et al., 2009; Ye et al., 2011). Our study was the first to directly compare the effect of emulsions covered by the singular interfacial component MPL and casein on the lipolysis rate based on exactly the same size of fat globules. The results indicated that the amounts of FFA released as well as the lipid hydrolysis rates were significantly different between the MPL and CN groups during gastric digestion. Compared with the droplets covered by MPL, the CN groups with a higher initial lipolysis rate soon reached a plateau with a lower FFA release amount during gastric digestion.

Intestinal Digestion. When the pregastric digested milk was mixed with simulated intestinal fluid and bile extracts, the interface of the droplets was immediately displaced by the bile salts. However, the initial absolute zeta-potentials of the $\mathrm{CN}$ groups were relatively lower, suggesting that the bile salts did not fully displace their interfacial components. This may explain why the initial rate of the $\mathrm{CN}$ groups was lower than that of the droplets covered by MPL. As the intestinal digestion proceed, the pancreatic lipase could hydrolyze the triglyceride molecules into FFA and monoglycerides, which would accumulate at the interface and decrease the zeta-potential. At the same time, the lipolytic products could be removed from the interface by being solubilized into mixed bile salt-phospholipid micelles and phospholipid vesicles (Gallier et al., 2012). Therefore, the zeta-potential of each emulsion remained fairly constant during the simulated intestinal digestion; however, the MPL groups still showed significant digestibility superiority over the $\mathrm{CN}$ groups during the later simulated intestinal digestion phase. Our results were similar to previous studies that compared the digestibility of processed fat globules and native globules (Berton et al., 2012; Garcia et al., 2014; de Oliveira et al., 2017). Although the homogenization of fat globules offers a larger available interfacial area, it would result in lipid droplets with the biochemical composition and architecture of the interfaces less favorable to the pancreatic lipase activity compared with the native fat globules covered by the MFGM (Berton et al., 2012). This may explain why droplets covered with MPL were digested more efficiently than those with casein.

\section{Effect of Droplet Size on In Vitro Infant Gastrointestinal Digestion}

Gastric Digestion. Concerning the effect of droplet size on the lipolysis of emulsions during gastric digestion, a significant difference was observed in the $\mathrm{CN}$ groups, with a limited effect of size on the lipolysis of droplets covered by MPL. Previous studies showed significantly different lipolysis levels between the small native milk fat globules and the large ones, mainly due
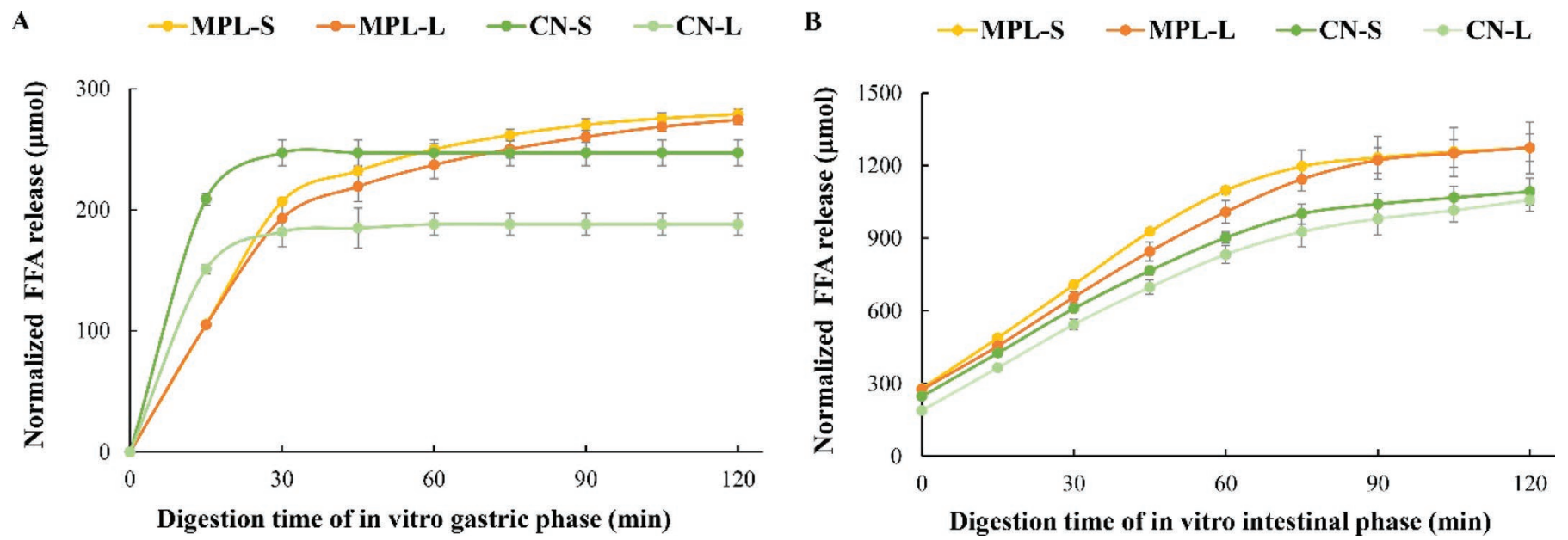

Figure 6. Total free fatty acid (FFA) release of droplets from different emulsions during in vitro gastric (A) and intestinal digestion (B). MPL-S and MPL-L indicate small and large globules covered with milk polar lipids, and CN-S and CN-L indicate small and large globules covered with casein, respectively. Error bars represent the SD of triplicate experiments. 
to the difference in specific surface area as well as the lipid composition (Garcia et al., 2014; Singh and Gallier, 2017). Although not entirely consistent, previous reports showed some differences in lipid composition between small and large native milk fat globules. Compared with larger globules, the smaller fat globules were richer in medium-chain fatty acids and myristic acid and poorer in long-chain fatty acids (Briard et al., 2003; Michalski et al., 2006; Lopez, 2011). Michalski et al. (2006) found the MFGM phospholipids of smaller fat globules contain less PC and SM, whereas Lopez (2011) reported a similar SM but higher PE concentration in smaller fat globules. Moreover, Mesilati-Stahy and Argov-Argaman (2014) found the differences in phosphatidylserine concentration between small and large fat globules were related to the lactation stages. The gastric lipase were found to selectively release short- and medium-chain fatty acids, because of their stereospecificity (Sams et al., 2016). Moreover, the adsorption of gastric lipase onto the emulsion interface could be reinforced with more negatively charged phospholipids, such as phosphatidylserine (Bourlieu et al., 2016). Therefore, the effect of the size of native milk fat globules on their lipolysis level during digestion reported in previous studies was again due to the intricate effects of size and interface composition. Our study was the first to directly compare the effect of the size of emulsions on lipolysis rate during gastric digestion based on exactly the same interfacial component MPL. The results indicated that, during gastric digestion, the size of droplets did not statistically affect the lipolysis of droplets covered with MPL, most probably due to the similar lipid composition of different-sized droplets.

Intestinal Digestion. During simulated intestinal digestion, small droplets showed a higher initial lipolysis rate and FFA release amount than the large ones, but the differences between different sizes narrowed with the digestion time. When pregastric digested milk fat globules enter the intestine, bile salt may replace some of the original emulsifiers and break down the original structure of droplets (Singh and Gallier, 2017), which might narrow the gap between different sizes of fat droplets.

\section{CONCLUSIONS}

Our study is the first to investigate the precise effect of singular interfacial components MPL of droplets on in vitro infant gastrointestinal digestion. Moreover, by mimicking the size of globules in human milk and infant milk formula, we also studied the effect of the size of these emulsions on gastrointestinal digestion. The MPL groups showed a lower initial lipolysis rate at the beginning of gastric digestion compared with the drop- lets covered by casein. However, droplets covered by MPL were superior to tose covered by $\mathrm{CN}$ during later digestion, with a significantly more stable droplet and a higher amount of FFA release. Smaller droplets generally showed a better digestibility than large droplets, with a higher initial lipolysis rate and amount of FFA release during gastrointestinal digestion. However, the effect of size on gastrointestinal digestion was relatively small compared with the interface composition, probably because the different sized droplets prepared in our study had similar lipid composition. Better knowledge of the digestibility of fat globules with different singular milk-derived interfacial compositions and sizes in the simulated in vitro infant gastrointestinal digestion will help contribute to the development of infant food with improved lipid digestion. Further work on the FFA release mode and the evolution of lipid and protein classes of droplets with singular interfacial composition during in vitro infant gastrointestinal digestion is underway in our laboratory.

\section{ACKNOWLEDGMENTS}

This work was supported by the Beijing $\mathrm{Mu}-$ nicipal Commission of Education Co-constructed program, Beijing Science and Technology Project (D141100004814001) and the 111 Project from the Education Ministry of China (No. B18053). We thank Edanz Group (China) for editing a draft of this manuscript. The authors declare no conflict of interest.

\section{REFERENCES}

Aken, G. A. V., E. V. D. Linden, J. Ubbink, and G. Duchateau. 2010. Relating food emulsion structure and composition to the way it is processed in the gastrointestinal tract and physiological responses: What are the opportunities? Food Biophys. 5:258-283.

Bénarouche, A., V. Point, G. Parsiegla, F. Carrière, and J. F. Cavalier. 2013. New insights into the $\mathrm{pH}$-dependent interfacial adsorption of dog gastric lipase using the monolayer technique. Colloids Surf. B Biointerfaces 111:306-312.

Berton, A., S. Rouvellac, B. Robert, F. Rousseau, C. Lopez, and I. Crenon. 2012. Effect of the size and interface composition of milk fat globules on their in vitro digestion by the human pancreatic lipase: Native versus homogenized milk fat globules. Food Hydrocoll. 29:123-134.

Bourlieu, C., O. Ménard, K. Bouzerzour, G. Mandalari, A. Macierzanka, A. R. Mackie, and D. Dupont. 2014. Specificity of infant digestive conditions: Some clues for developing relevant in vitro models. Crit. Rev. Food Sci. Nutr. 54:1427-1457.

Bourlieu, C., O. Ménard, L. C. A. De, L. Sams, F. Rousseau, M. N. Madec, B. Robert, A. Deglaire, S. Pezennec, and S. Bouhallab. 2015. The structure of infant formulas impacts their lipolysis, proteolysis and disintegration during in vitro gastric digestion. Food Chem. 182:224-235.

Bourlieu, C., G. Paboeuf, S. Chever, S. Pezennec, J. F. Cavalier, F. Guyomarc'h, A. Deglaire, S. Bouhallab, D. Dupont, F. Carrière, and V. Vie. 2016. Adsorption of gastric lipase onto multicomponent model lipid monolayers with phase separation. Colloids Surf. B Biointerfaces 143:97-106. 
Briard, V., N. Leconte, F. Michel, and M.-C. Michalski. 2003. The fatty acid composition of small and large naturally occurring milk fat globules. Eur. J. Lipid Sci. Technol. 105:677-682.

Chahinian, H., T. Snabe, C. Attias, P. Fojan, S. B. Peterson, and F. Carrière. 2006. How gastric lipase, an interfacial enzyme with a Ser-His-Asp catalytic triad, acts optimally at acidic pH. Biochemistry 45:993-1001.

Chinese Standard. 2016. National food safety standard for the determination of fatty acids in food. GB 5009.168-2016. National Health and Family Planning Commission of the People's Republic of China, China Food and Drug Administration, Beijing, China.

Cornec, M., P. J. Wilde, P. A. Gunning, A. R. Mackie, F. A. Husband, M. L. Parker, and D. C. Clark. 2010. Emulsion stability as affected by competitive adsorption between an oil-soluble emulsifier and milk proteins at the interface. J. Food Sci. 63:39-43.

de Oliveira, S. C., A. Bellanger, O. Ménard, P. Pladys, G. Y. Le, E. Dirson, F. Kroell, D. Dupont, A. Deglaire, and C. Bourlieu. 2017. Impact of human milk pasteurization on gastric digestion in preterm infants: a randomized controlled trial. Am. J. Clin. Nutr. 105:379-390.

Gallier, S., D. Acton, M. Garg, and H. Singh. 2017. Natural and processed milk and oil body emulsions: Bioavailability, bioaccessibility and functionality. Food Struct. 13:13-23.

Gallier, S., J. Cui, T. D. Olson, S. M. Rutherfurd, A. Ye, P. J. Moughan, and H. Singh. 2013a. In vivo digestion of bovine milk fat globules: effect of processing and interfacial structural changes. I. Gastric digestion. Food Chem. 141:3273-3281.

Gallier, S., K. Vocking, J. A. Post, D. H. B. Van, D. Acton, V. D. B. Em, and B. T. Van. 2015. A novel infant milk formula concept: Mimicking the human milk fat globule structure. Colloids Surf. B Biointerfaces 136:329-339.

Gallier, S., A. Ye, and H. Singh. 2012. Structural changes of bovine milk fat globules during in vitro digestion. J. Dairy Sci. 95:35793592.

Gallier, S., X. Q. Zhu, S. M. Rutherfurd, A. Ye, P. J. Moughan, and H. Singh. 2013b. In vivo digestion of bovine milk fat globules: Effect of processing and interfacial structural changes. II. Upper digestive tract digestion. Food Chem. 141:3215-3223.

Garcia, C., C. Antona, B. Robert, C. Lopez, and M. Armand. 2014. The size and interfacial composition of milk fat globules are key factors controlling triglycerides bioavailability in simulated human gastro-duodenal digestion. Food Hydrocoll. 35:494-504.

Golding, M., and T. J. Wooster. 2010. The influence of emulsion structure and stability on lipid digestion. Curr. Opin. Colloid Interface Sci. 15:90-101.

Golding, M., T. J. Wooster, L. Day, M. Xu, L. Lundin, J. Keogh, and P. Clifton. 2011. Impact of gastric structuring on the lipolysis of emulsified lipids. Soft Matter 7:3513-3523.

Li, Y., H. Mu, A. Jensenevoldthaulov, X. Xu, M. Otto, and O. Anne. 2010. New human milk fat substitutes from butterfat to improve fat absorption. Food Res. Int. 43:739-744.

Lopez, C. 2011. Milk fat globules enveloped by their biological membrane: Unique colloidal assemblies with a specific composition and structure. Curr. Opin. Colloid Interface Sci. 16:391-404.

Luo, J., Z. Huang, H. Liu, Y. Zhang, and F. Ren. 2018. Yak milk fat globules from the Qinghai-Tibetan Plateau: Membrane lipid composition and morphological properties. Food Chem. 245:731-737.

Maldonado-Valderrama, J., P. Wilde, A. Macierzanka, and A. Mackie 2011. The role of bile salts in digestion. Adv. Colloid Interface Sci. 165:36-46.
Mandalari, G., K. Adelpatient, V. Barkholt, C. Baro, L. Bennett, M. Bublin, S. Gaier, G. Graser, G. S. Ladics, and D. Mierzejewska. 2009. In vitro digestibility of $\beta$-casein and $\beta$-lactoglobulin under simulated human gastric and duodenal conditions: A multi-laboratory evaluation. Regul. Toxicol. Pharmacol. 55:372-381.

Mesilati-Stahy, R., and N. Argov-Argaman. 2014. The relationship between size and lipid composition of the bovine milk fat globule is modulated by lactation stage. Food Chem. 145:562-570.

Michalski, M. C., N. Leconte, V. Briard-Bion, J. Fauquant, J. Maubois, and H. Goudedranche. 2006. Microfiltration of raw whole milk to select fractions with different fat globule size distributions: Process optimization and analysis. J. Dairy Sci. 89:3778-3790.

Michalski, M. C. 2009. Specific molecular and colloidal structures of milk fat affecting lipolysis, absorption and postprandial lipemia. Eur. J. Lipid Sci. Technol. 111:413-431.

Michalski, M.-C., F. Michel, D. Sainmont, and V. Briard. 2002. Apparent $\zeta$-potential as a tool to assess mechanical damages to the milk fat globule membrane. Colloids Surf. B Biointerfaces 23:23-30.

Oosting, A., D. Kegler, H. J. Wopereis, I. C. Teller, B. J. M. V. D. Heijning, H. J. Verkade, and E. M. V. D. Beek. 2012. Size and phospholipid coating of lipid droplets in the diet of young mice modify body fat accumulation in adulthood. Pediatr. Res. 72:362-369.

Phan, T. T. Q., T. T. Le, D. V. D. Walle, P. V. D. Meeren, and K. Dewettinck. 2016. Combined effects of milk fat globule membrane polar lipids and protein concentrate on the stability of oil-in-water emulsions. Int. Dairy J. 52:42-49.

Roman, C., F. Carriere, P. Villeneuve, M. Pina, V. Millet, U. Simeoni, and J. Sarles. 2007. Quantitative and qualitative study of gastric lipolysis in premature infants: Do MCT-enriched infant formulas improve fat digestion? Pediatr. Res. 61:83-88.

Sams, L., J. Paume, J. Giallo, and F. Carrière. 2016. Relevant pH and lipase for in vitro models of gastric digestion. Food Funct. 7:30-45.

Schipper, L., D. G. Van, L. M. Broersen, M. Loos, N. Bartke, A. J. Scheurink, and V. D. B. Em. 2016. A postnatal diet containing phospholipids, processed to yield large, phospholipid-coated lipid droplets, affects specific cognitive behaviors in healthy male mice. J. Nutr. 146:1155-1161.

Shimizu, M., K. Yamauchi, and C. Kanno. 1980. Effect of proteolytic digestion of milk fat globule membrane proteins on stability of the globules. Milchwissenschaft 35:9-12.

Singh, H., and S. Gallier. 2017. Nature's complex emulsion: The fat globules of milk. Food Hydrocoll. 68:81-89.

Spitsberg, V. L. 2005. Invited review: Bovine milk fat globule membrane as a potential nutraceutical. J. Dairy Sci. 88:2289-2294.

Vanderghem, C., P. Bodson, S. Danthine, M. Paquot, C. Deroanne, and C. Blecker. 2010. Milk fat globule membrane and buttermilks: From composition to valorization. Biotechnol. Agron. Soc. Environ. 14:354-362

Vanderghem, C., F. Francis, S. Danthine, C. Deroanne, M. Paquot, E. De Pauw, and C. Blecker. 2011. Study on the susceptibility of the bovine milk fat globule membrane proteins to enzymatic hydrolysis and organization of some of the proteins. Int. Dairy J. $21: 312-318$.

Ye, A., J. Cui, and H. Singh. 2011. Proteolysis of milk fat globule membrane proteins during in vitro gastric digestion of milk. J. Dairy Sci. 94:2762. 